\title{
Absolute Position and Energy
}

\author{
Eyal Brodet ${ }^{1}$ \\ ${ }^{1}$ Michaeslon St, Jerusalem, 93707, Israel \\ Correspondence: Eyal Brodet, Michaeslon St, Jerusalem, 93707, Israel. E-mail: eyalbrodet@hotmail.com \\ Received: June 10, 2018 \\ Accepted: June 19, 2018 \\ Online Published: July 9, 2018 \\ doi:10.5539/apr.v10n4p1 \\ URL: https://doi.org/10.5539/apr.v10n4p1
}

\begin{abstract}
In this paper we will develop further the absolute position of a particle defined in Brodet $(2017 \mathrm{a}, \mathrm{b})$ which involves the particle's decay time or when relevant internal time and the particle's velocity with respect to the expanding universe. We will refine the previous definition to give two separate absolute position definitions, one termed the absolute position at rest and the other termed the absolute position which includes also a contribution of the particle's velocity with respect to the velocity of the expanding universe. Next we will discuss how we may define the particle's absolute energy from the particle's absolute position definition. We will show, how the absolute energy definition may help us to identify a dependence between the particle's decay time, as measured in its rest frame, and its velocity with respect to the expanding universe. Consequently, we will relate the above to the particle's mean lifetime and discuss the affect and relationship of the running coupling constant and the possible mean lifetime dependence on velocity. Finally, experimental ways by which one may investigate and test the above are presented.
\end{abstract}

Keywords: Absolute, position, energy, decay time, velocity

\section{Introduction}

In this paper we will develop further the absolute position definition given in Brodet (2017a, b) which uses the particle's decay time or when relevant internal time and the particle's velocity with respect to the expanding universe. We will refine the previous definition and get two separate definitions for the particle's absolute position: the first an absolute position that depends only on the particle internal properties and doesn't depend on velocity, and the second an absolute position that additionally includes a contribution from the particle's velocity with respect to the velocity of the expanding universe. Next, we will show how one may obtain the particle's absolute energy from the particle's absolute position definition. We will also show, how the absolute energy expression may help us identify a dependence between the particle's decay time at its rest frame and the particle's velocity with respect to the expanding universe. Consequently, we will relate the above to the particle's mean lifetime and discuss the affect and relationship of the running coupling constant and the possible mean lifetime dependence on velocity. Finally, experimental ways to investigate and test the above are presented.

This paper is divided into six sections. Section 2 discusses the absolute position of the particle that doesn't depend on velocity. Section 3 discusses the absolute position of the particle that depends on velocity and the possible consequence of the particle absolute energy. Section 4 discusses the particle's absolute energy at rest and section 5 discusses the experimental implications. Section 6 contains the conclusions.

\section{Absolute Position that does not Depend on Velocity}

The absolute position, $P_{i(a b s)}^{\prime}$, is defined in Brodet (2017a) as:

$$
P_{i(a b s)}^{\prime}=\left(V_{i\left(T\left(V_{r}\right)\right)} t_{i}\right)^{2}=\frac{M_{i}}{t_{M_{i(\text { mean })}}}\left(\frac{\left(V_{r(i)(T)} t_{i}\right)^{2}}{2}+\frac{X_{i(a r b)}^{2}}{2}-\frac{\left(c_{i\left(V_{r(T))}^{\prime}\right.}^{\prime} t_{i}\right)^{2}}{2}\right)
$$

Where $t_{i}$ is particle's i decay time(or internal time in the case of a stable particle), $c_{i\left(V_{r(T)}^{\prime}\right)}^{\prime}$ is the particle's internal velocity defined in Brodet (2017b), $V_{r(i)(T)}$ is the particle's velocity with respect to the expanding universe, 
$V_{i\left(T\left(V_{r(T)}\right)\right)}$ is the total particle's velocity, $X_{i(\text { arb })}$ is the particle's position in some arbitrary frame of reference and $t_{M_{i(\text { mean })}}=\frac{h_{i(\text { mean })}}{M_{i(\text { mean })} c_{i(m e a n)}^{2}}$ as defined in Brodet (2018).

Choosing a frame in which $X_{i(a r b)}=0$ gives:

$P_{i(\text { abs })}^{\prime}=\left(V_{i\left(T\left(V_{r}\right)\right)} t_{i}\right)^{2}=\frac{M_{i}}{t_{M_{i(\text { mean })}}}\left(\frac{\left(V_{r(i)(T)} t_{i}\right)^{2}}{2}-\frac{\left(c_{i\left(V_{r(T))}\right.} t_{i}\right)^{2}}{2}\right)$

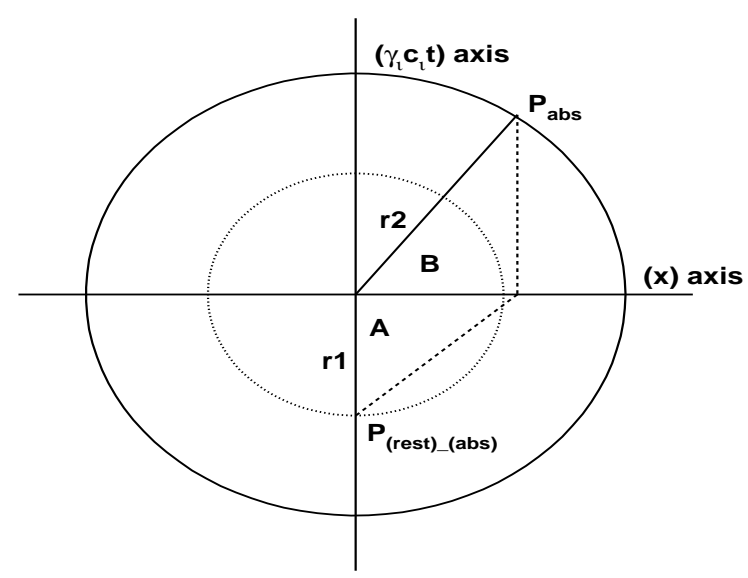

Figure 1. The absolute position, $P_{i(a b s)}$, and the restl absolute position, $P_{i(r e s t)_{-}(a b s)}$

Turning into the geometric picture of Fig. 1 in which we insist that $V_{i\left(T\left(V_{r(T)}\right)\right)}$ or the radius r1 in triangle A, wouldn't depend on $V_{r(i)(T)}$, such $V_{i(T)}=c_{i(0)}^{\prime}$, (and temporarily removing the $\frac{M_{i}}{t_{M_{i(m e a n)}}}$ factor) we get:

$$
-\left(\sqrt{2} c_{i(0)}^{\prime} t_{i}\right)^{2}=A\left(V_{r(i)(T)} t_{i}\right)^{2}-\mathrm{B}\left(c_{i\left(V_{i(r)(T)}\right.} t_{i}\right)^{2}
$$

Where $c_{i}$ and $c_{i(0)}$ may be given by Brodet $(2017 \mathrm{~b}, 2018)$ :

Or may be given by:

$$
\begin{gathered}
c_{i}=\frac{{ }^{c+\frac{a 1}{f_{r(i)}}}}{b 1\left(M_{i(\text { mean })}, V_{i(r)(T),}, A_{i}\right)} \\
c_{i(0)}^{\prime}=\frac{{ }^{c+\frac{a 1}{f_{r(i)}}}}{b 1\left(M_{i(\text { mean })}, A_{i}\right)}
\end{gathered}
$$

$$
\begin{gathered}
c_{i}=\frac{c+a 1 \cdot f_{r(i)}}{b 1\left(M_{\left.i(\text { mean }), V_{i(r)(T)}, A_{i}\right)}\right.} \\
c_{i(0)}^{\prime}=\frac{c+a 1 \cdot f_{r(i)}}{b 1\left(M_{i(\text { mean })}, A_{i}\right)}
\end{gathered}
$$

It is unclear yet if $c_{i}, c_{i(0)}^{\prime}$ should take the form of equations $3 \mathrm{a}, 3 \mathrm{~b}$ or $3 \mathrm{c}, 3 \mathrm{~d}$. It is possible that one set of equations is suitable for particles and the other to anti-particles. This should be determined experimentally as will be discussed in section 5 and in Brodet (2016, 2017a, 2017b, 2018). 
Try if it can be solved assuming that $\mathrm{A}=\mathrm{B}$

i.e. where $\sqrt{A}$ could be equal to:

$$
\left(\frac{-\left(\sqrt{2} c_{i(0)}^{\prime} t_{i}\right)^{2}}{-\left(c_{i}^{2}-V_{i(r)(T)}^{2}\right)}\right)=A t_{i}^{2}
$$

$$
\sqrt{A}=\gamma_{i}=\frac{1}{\sqrt{\frac{c_{i\left(V_{r(i)(T)}^{2}\right)}^{c_{i(0)}^{2}}-\frac{V_{i(r)(T)}^{2}}{c_{i(0)}^{2}}}{n^{2}}}}
$$

Where $c_{i(0)}^{2}=2 c_{i(0)}^{2 \prime}$

Finally, based on equations 1a,4a,b, we may define an absolute position for particle $\mathrm{i}$ that doesn't depend on its velocity and external position and depends only on its internal properties. Therefore, the above may be termed internal absolute position or rest absolute position, $P_{i(r e s t)_{-}(a b s)}$, given by (choosing $X_{i(a r b)}=0$ ):

$$
P_{i(\text { rest })_{-}(a b s)}=\frac{M_{i}}{t_{M_{i(\text { mean })}}}\left(\frac{\left(V_{r(i)(T)} \gamma_{i} t_{i}\right)^{2}}{2}-\frac{\left(c_{\left.i\left(V_{i(r)(T)}\right) \gamma_{i} t_{i}\right)^{2}}\right.}{2}\right)
$$

Equation 5a will be modified further later on in section 4. Notwithstanding, we may define from the above, an absolute four-vector:

$$
P 4_{i(\text { rest })_{-}(a b s)}=\left(\sqrt{\frac{M_{i}}{t_{M_{i(\text { mean })}}}} \frac{\gamma_{i} X_{i}}{\sqrt{2}}, \sqrt{\frac{M_{i}}{t_{M_{i(\text { mean })}}}} \frac{\gamma_{i} c_{i} t_{i}}{\sqrt{2}}\right)
$$

Which has a constant length of: $\sqrt{\frac{M_{i}}{t_{M_{i(\text { mean })}}}\left(c_{i(0)}^{\prime} t_{i}\right)^{2}}$

Since $\sqrt{\frac{M_{i}}{t_{M_{i(\text { mean })}}}}=\sqrt{M_{i}} \times \sqrt{\frac{M_{i(\text { mean })} c_{i(\text { mean })}^{2}}{h_{i(\text { mean })}}} \approx M_{i}$, (assuming $c_{i}=h_{i}=1$ ), we may modify Equation 5b to be consistent with special relativity arbitrary position four-vector( by changing $X_{i}$ to an arbitrary position $X_{i(a r b)}$ and using $c_{i\left(V_{r}\right)}$ that depends on $V_{i(r)}$ instead of $\left.V_{i(r)(T)}\right)$ such:

$$
P 4_{i(a r b)_{-}(s r)}=\left(M_{i} \gamma_{i} X_{i(a r b)}, M_{i} \gamma_{i} c_{i} t_{i}\right)
$$

Which defines an arbitrary but invariant length of $\sqrt{P P_{(a r b)_{s r}}}$, while temporarily removing the mass factor, $M_{i}$, and considering its position with respect to a frame in which $\gamma_{i}=1$, we get a consisted expression to special relativity, such:

$$
P 4_{i(a r b)_{-}(s r 1)}=X_{i(a r b)}^{2}-c_{i}^{2} t_{i}^{2}
$$

The values of $X_{i(a r b)}, t_{i}$ may be viewed by a frame of reference traveling in velocity $V_{r}$ with respect to the frame of $X_{i(a r b)}, t_{i}$ by using special relativity modified Lorentz transformation based on Brodet (2016):

Where

$$
X_{i(a r b)}^{c_{i} t_{i}^{\prime}}=\left(\begin{array}{cc}
\alpha_{i} \gamma_{i} & -\gamma_{i} \beta_{i} \\
-\gamma_{i} \beta_{i} & \alpha_{i} \gamma_{i}
\end{array}\right)^{c_{i} t_{i}}
$$

$$
\begin{aligned}
\gamma_{i} & =\frac{1}{\sqrt{\frac{c_{i\left(V_{r(i)(T)}^{2}\right.}^{c_{i(0)}^{2}}-\frac{V_{i(r)}^{2}}{c_{i(0)}^{2}}}{\sqrt{\alpha_{i}^{2}-\beta_{i}^{2}}}}} \\
& =>\gamma_{i}=\frac{1}{\sqrt{\alpha^{2}}}
\end{aligned}
$$

and $\beta_{i}=\frac{V_{r}}{c_{i(0)}}, \alpha_{i}=\frac{c_{i\left(V_{i(r)(T)}\right)}}{c_{i(0)}}$

Similarly, the transformation of the absolute position vector $P 4_{i(r e s t) \_(a b s)}$ may be performed by: 


$$
\begin{gathered}
c_{i} t_{i}^{\prime} / \sqrt{2} \\
X_{i}^{\prime} / \sqrt{2}
\end{gathered}=\left(\begin{array}{cc}
\alpha_{i}^{\prime} \gamma_{i}^{\prime} & -\gamma_{i}^{\prime} \beta_{i}^{\prime} \\
-\gamma_{i}^{\prime} \beta_{i}^{\prime} & \alpha_{i}^{\prime} \gamma_{i}^{\prime}
\end{array}\right) \gamma_{i} c_{i} t_{i} / \sqrt{2}
$$

Where $\gamma_{i}=\frac{1}{\sqrt{\frac{c_{i\left(V_{r(i)(T)}\right.}^{c_{i(0)}^{2}}-\frac{V_{i(r)(T)}^{2}}{c_{i(0)}^{2}}}{2}}}$

and $\beta_{i}=\frac{V_{r}(T)}{c_{i(0)}}, \quad \alpha_{i}=\frac{c_{i\left(V_{i(r)(T)}\right)}}{c_{i(0)}}$

and

and

$$
\begin{gathered}
\gamma_{i}^{\prime}=\frac{1}{\sqrt{\frac{c_{i\left(V_{r(i)(T)}^{\prime}\right)}^{c_{i(0)}^{2}}-\frac{V_{i(r)(T)}^{2 \prime}}{c_{i(0)}^{2}}}{\beta_{i}}}} \\
\beta_{i}=\frac{V_{i(r)(T)}^{\prime}}{c_{i(0)}}, \alpha_{i}^{\prime}=\frac{c_{i\left(V_{r(i)(T)}^{\prime}\right)}^{\prime}}{c_{i(0)}}
\end{gathered}
$$

A more complete transformation of a position four-vector as defined above will be discussed later on in the text.

\section{Absolute Position that Depends on Velocity}

The total absolute position of the particle should depend on its internal properties but also on the particle's velocity and therefore describes the sum of the space and time contributions. The above is related to the discussion in Brodet (2017) where initially the absolute of the complex number $z=X_{i}+i c_{i} t_{i}$ is given by $|z|=\sqrt{X_{i}^{2}+c_{i}^{2} t_{i}^{2}}$. Since, in this case, we are interested in including the contribution of the particle's velocity to its absolute position definition, we adopt the plus sign for the time contribution such:

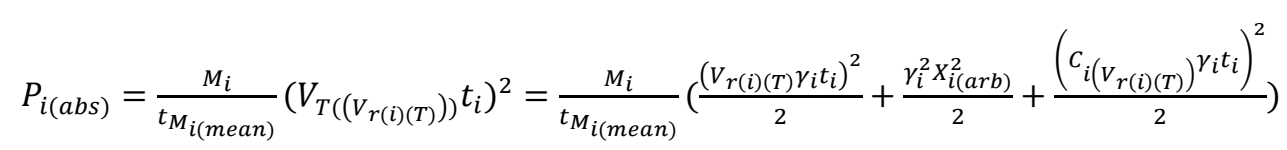

The geometric meaning of $P_{i(a b s)}$ is shown in Figure 1 with respect to the geometrical meaning of $P_{i(r e s t)_{-}(a b s)}$. Choosing $X_{i(a r b)}^{2}=0$ gives:

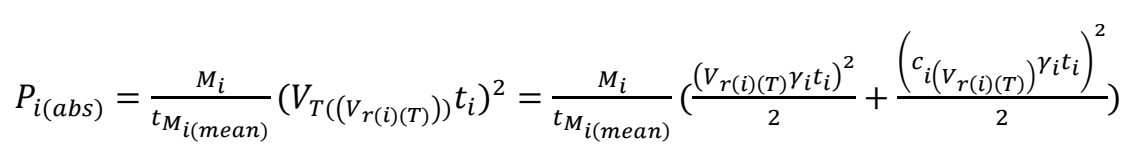

One can see that we actually get the time and position components at the end of the universe frame of reference (i.e. a frame resting on the edge of the expanding universe), which could be used as an absolute frame of reference. Therefore, we may define:

Where

$$
\begin{gathered}
t_{i(a b s)}=\gamma_{i} t_{i} \\
X_{i(a b s)}=V_{r(i)(T)} t_{i(a b s)}
\end{gathered}
$$

$$
\gamma_{i}=\frac{1}{\sqrt{\alpha_{i}^{2}-\beta_{i}^{2}}}
$$

Which allows us to express $P_{i(a b s)}$ in terms of $t_{i(a b s)}, X_{i(a b s)}$ :

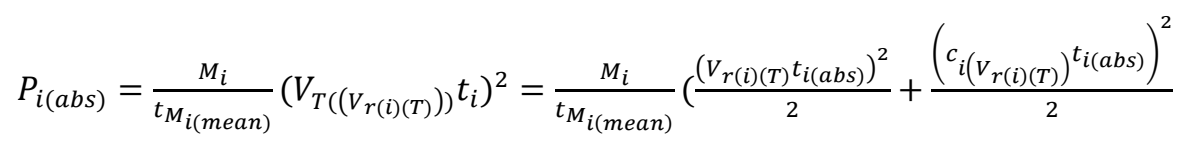




$$
P_{i(a b s)}=\frac{M_{i}}{t_{M_{i(\text { mean })}}}\left(V_{T\left(\left(V_{r}\right)(i)(T)\right)} t_{i}\right)^{2}=\frac{M_{i}}{t_{M_{i(\text { mean })}}}\left(\frac{\left(X_{i(a b s)}\right)^{2}}{2}+\frac{\left(c_{i\left(V_{r(i)(T)}\right)} t_{i(a b s)}\right)^{2}}{2}\right)
$$

Taking the total derivative may give the absolute angular momentum of particle i such:

$$
\Delta P_{i(a b s)}=\frac{\partial P_{i(a b s)}}{\partial X_{i(a b s)}} \Delta X_{i(a b s)}+\frac{\partial P_{i(a b s)}}{\partial t_{i(a b s)}} \Delta t_{i(a b s)}
$$

where

$$
\frac{\partial P_{i(a b s)}}{\partial X_{i(a b s)}}=P_{i(a b s)}=\frac{M_{i}}{t_{M_{i(m e a n)}}} X_{i(a b s)}=\frac{M_{i}}{t_{M_{i(m e a n)}}} \gamma_{i} V_{r(i)(T)} t_{i}
$$

and

$$
\frac{\partial P_{i(a b s)}}{\partial t_{i(a b s)}}=E_{i(a b s)}=\frac{M_{i}}{t_{M_{i(m e a n)}}} c_{i\left(V_{r(i)(T)}^{2}\right.} t_{i(a b s)}=\gamma_{i} \frac{M_{i} c_{i\left(V_{r(i)(T)}^{2}\right.}}{t_{M_{i(\text { mean })}}} t_{i}
$$

Where $E_{i(a b s)}, P_{i(a b s)}$ are the total absolute energy and momentum of particle i.

Therefore:

$$
\begin{aligned}
& \Delta P_{i(a b s)}=L_{i(a b s)}=\frac{M_{i}}{t_{M_{i(\text { mean })}}} \gamma_{i} X_{i} \Delta X_{i(a b s)}+\frac{M_{i}}{t_{M_{i(m e a n)}}} \gamma_{i} c_{i\left(V_{r(i)(T)}\right.} t_{i} \Delta t_{i(a b s)} \\
& \Delta P_{i(a b s)}=L_{i(a b s)}=\frac{M_{i}}{t_{M_{i(m e a n)}}} \gamma_{i} V_{r(i)(T)} t_{i} \Delta X_{i(a b s)}+\frac{M_{i}}{t_{M_{i(m e a n)}}} \gamma_{i} c_{i\left(V_{r(i)(T)}\right.} t_{i} \Delta t_{i(a b s)} \\
& \Delta P_{i(a b s)}=L_{i(a b s)}=\left(P_{i(a b s)} \Delta X_{i(a b s)}+E_{i(a b s)} \Delta t_{i(a b s)}\right)
\end{aligned}
$$

Which may be used to define particle's i absolute energy by:

$$
E_{i(a b s)}=\frac{L_{i(a b s)}}{t_{i(a b s)}}=\frac{L_{i(a b s)}}{\gamma_{i} t_{i}}=\left(\frac{M_{i}}{t_{M_{i(m e a n)}}} \gamma_{i} V_{r(i)(T)} \Delta X_{i(a b s)}+\frac{M_{i}}{t_{M_{i(m e a n)}}} \gamma_{i} c_{i\left(V_{r(i)(T)}\right.}^{2} \Delta t_{i(a b s)}\right)
$$

Which should be equal to our former expression for particle's i absolute energy given in Equation 11c:

$$
E_{i(a b s)}=\gamma_{i} \frac{M_{i} c_{i\left(V_{r(i)(T)}^{2}\right.}}{t_{M_{i(\text { mean })}}} t_{i}
$$

Such:

Therefore:

$$
\frac{M_{i}}{t_{M_{i(\text { mean })}}}\left(V_{r(i)(T)} \Delta X_{i(a b s)}+c_{i\left(V_{r(i)(T)}^{2}\right.}^{2} \Delta t_{i(a b s)}\right)=\gamma_{i} \frac{M_{i} c_{i\left(V_{r(i)(T)}\right.}^{2}}{t_{M_{i(\text { mean })}}} t_{i}
$$

$$
\left(V_{r(i)(T)} \Delta X_{i(a b s)}+c_{i\left(V_{r(i)(T)}^{2}\right.} \Delta t_{i(a b s)}\right)=\gamma_{i} c_{i\left(V_{r(i)(T)}^{2}\right.} t_{i}
$$

Let:

$$
\begin{aligned}
& \Delta t_{i(a b s)}=\gamma_{i} t_{i(0)} \\
& \Delta X_{i(a b s)}=V_{r(i)(T)} \gamma_{i} t_{i(0)}
\end{aligned}
$$

Therefore:

Let:

$$
\begin{aligned}
& \gamma_{i}\left(V_{r(i)(T)}^{2} \gamma_{i} t_{i(0)}+c_{i\left(V_{r(i)(T)}\right.}^{2} \gamma_{i} t_{i(0)}\right)=\gamma_{i} c_{i\left(V_{r(i)(T)}\right.}^{2} t_{i} \\
& \gamma_{i} c_{i\left(V_{r(i)(T)}\right.}^{2}\left(\frac{V_{r(i)(T)}^{2}}{c_{i\left(V_{r(i)(T)}\right.}^{2}} t_{i(0)}+t_{i(0)}\right)=\gamma_{i} c_{i\left(V_{r(i)(T)}^{2}\right.}^{2} t_{i}
\end{aligned}
$$

$$
\beta_{i}^{2}=\frac{V_{r(i)(T)}^{2}}{c_{i\left(V_{r(i)(T)}^{2}\right.}^{2}} t_{i(0)}
$$




$$
\begin{aligned}
& \gamma_{i} c_{i\left(V_{r(i)(T)}^{2}\right.}\left(\beta_{i}^{2} t_{i(0)}+t_{i(0)}\right)=\gamma_{i} c_{i\left(V_{r(i)(T)}^{2}\right.} t_{i} \\
& t_{i}=t_{i(0)}+\beta_{i}^{2} t_{i(0)}=t_{i(0)}\left(1+\beta_{i}^{2}\right)
\end{aligned}
$$

Therefore, in continuation to the discussion in Brodet (2017) about the effect of the running coupling constant on the particle's decay time:

Where

$$
\begin{gathered}
\gamma_{i} t_{i}=\gamma_{i}\left(t_{i(0)}+\frac{V_{i(r)(T)}^{2}}{c_{i(0)}^{2}} t_{i(0)}\right)=\gamma_{i} t_{i(0)}\left(1+\beta_{i\left(V_{i(r)(T)}^{2}\right)}^{2}\right) \\
\gamma_{i} t_{i}=\gamma_{i(\text { new })} t_{i(0)}
\end{gathered}
$$

$$
\gamma_{i(\text { new })}=\gamma_{i}\left(1+\beta_{i\left(V_{i(r)(T)}\right)}^{2}\right)
$$

Where

$$
\gamma_{i}=\frac{1}{\sqrt{\frac{c_{i\left(V_{i(r)(T)}\right)}^{c_{i(0)}^{2}}-\frac{V_{i(r)(T)}^{2}}{c_{i(0)}^{2}}}{2}}}
$$

Connecting $t_{i(0)}$ to the hidden variable in time $f_{r}$ (Brodet, 2010) and considering a particle at rest (i.e. $V_{i(r)(T)}=$ $V_{\text {exp }}$ where $V_{\text {exp }}$ describes the velocity of the particle with respect to the expanding universe), gives:

$$
\begin{gathered}
t_{i(0)}+\frac{V_{(\text {exp })}^{2}}{c_{i 0)}^{2}} t_{i(0)}=t_{i(0)}\left(1+\beta_{i(\exp )}^{2}\right)=\tau_{M_{i}} \ln \left(\frac{f\left(M_{i}\right)}{f_{r}}\right) \\
t_{i(0)}=\frac{\tau_{M_{i}} \ln \left(\frac{f\left(M_{i}\right)}{f_{r}}\right)}{\left(1+\beta_{i(\text { exp })}^{2}\right)}
\end{gathered}
$$

Including the possible effect of particle's i potential energy, according to Brodet $(2012,2013,2018)$ gives a possible expression for particle's i decay time, $t_{i}$ such:

$$
t_{i}=\frac{c 1 \tau_{M_{i}} l n\left(\frac{f\left(M_{i}\right)}{f_{r}}\right)}{\left(1+\beta_{i(\text { exp })}^{2}\right)}\left(1+\beta_{i}^{2}\right)+\frac{c 2}{f_{\text {potential }}}
$$

Where $f_{\text {potential }}$ for the electric potential may be given by Brodet (2012):

$$
f_{\text {potential }(\text { elec })}=\left|\frac{k Q_{i} Q_{j}}{r_{i j}}\right|
$$

Where $Q_{i}$ and $Q_{j}$ are the electric charges of particle's i and $\mathrm{j}$, and $r_{i j}$ is the distance between particle's $\mathrm{i}$ and $\mathrm{j}$, and $\beta_{\text {exp }}=\frac{V_{\text {exp }}}{c_{i(0)}}$.

As discussed in Brodet (2017b), it may be possible to get the general charge of particle i by deriving the expression for the particle's general angular momentum with respect to $f_{r}$. Therefore, we may derive our new expression for the particle's absolute angular momentum, $L_{i(a b s)}$, and get particle's i absolute total charge, $Q_{i(T)_{(}(a b s)}$,such:

And,

$$
L_{i(\text { abs })}=\gamma_{i}^{2}\left(\frac{M_{i}}{t_{M_{i(\text { mean })}}} V_{r(i)(T)}^{2}\left(\frac{\tau_{M_{i}} \ln \left(\frac{f\left(M_{i}\right)}{f_{r}}\right)}{\left(1+\beta_{i(\text { exp })}^{2}\right)}\right)^{2}\left(1+\beta_{i}^{2}\right)+\frac{M_{i}}{t_{M_{i(\text { mean })}}} c_{i\left(V_{r(i)(T)}\right.}^{2}\left(\frac{\tau_{M_{i}} \ln \left(\frac{f\left(M_{i}\right)}{f_{r}}\right)}{\left(1+\beta_{i(\text { exp })}^{2}\right)}\right)^{2}\left(1+\beta_{i}^{2}\right)\right)
$$

$$
\frac{\partial L_{i(a b s)}}{\partial f_{r}}=Q_{i(T)_{-}(a b s)}
$$

The resulted derivative is trivial but as it is a long expression so it will not be shown here. It is suggested that the derivative $\frac{\partial L_{i(a b s)}}{\partial f_{r}}$ of the absolute angular momentum shown in equation $18 \mathrm{~b}$, gives a more accurate value for particle's i absolute total charge $Q_{i(T)_{-}(a b s)}$, than the expression given earlier in Brodet (2017b). The value of 
$Q_{i(T)_{(}(a b s)}$ given here, describes the dependence of the particle's charge on the value of the particle's velocity $V_{r(T)}$ and therefore may give the complete dependency of the charge on the velocity (and hence the energy), which may contain and explain the already known dependency of the charge on velocity through the running coupling constant effect (Halzen \& Martin, 1984). In particular, the dependence of $t_{i}$ on $V_{r(T)}$ which is included in the expression for $Q_{i(T)_{-}(a b s)}$ may be related to the running constant effect as will be discussed in section 5 .

Therefore, applying the Lorentz transformation of equation $7 \mathbf{c}$, to the absolute four-vector, $P 4_{i(r e s t)_{-}(a b s)}$, we get:

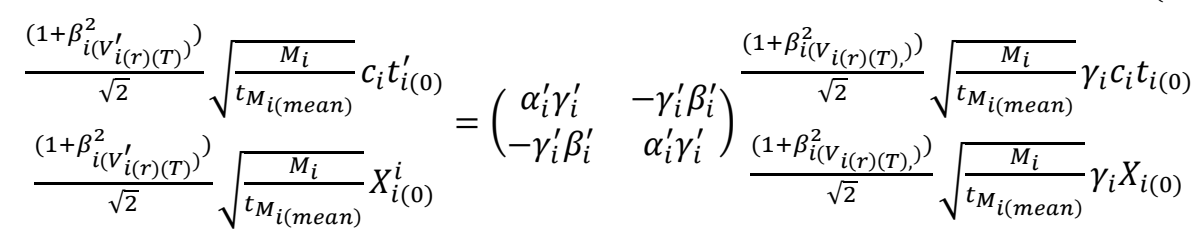

Where

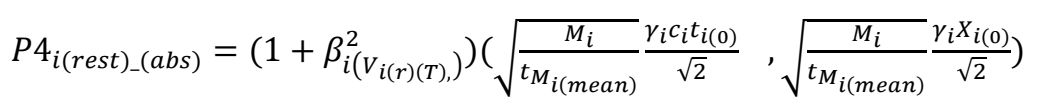

and $\gamma_{i}^{\prime}, \alpha_{i}^{\prime}, \beta_{i}^{\prime}$ are given in equation $7 \mathrm{~d}, P 4_{i(r e s t)_{(}(a b s)}^{\prime}$ is how the absolute vector, $P 4_{i(r e s t)_{-}(a b s)}$, is viewed if it is moving with velocity $V_{r}^{\prime}$ with respect to an observer:

$$
P 4_{i(r e s t)_{-}(a b s)}^{\prime}=\left(1+\beta_{i\left(V_{i(r)(T)}^{\prime}\right)}^{2}\right)\left(\sqrt{\frac{M_{i}}{t_{M_{i(\text { mean })}}}} \frac{c_{i} t_{i(0)}^{\prime}}{\sqrt{2}}, \sqrt{\frac{M_{i}}{t_{M_{i(\text { mean })}}}} \frac{x_{i(0)}^{\prime}}{\sqrt{2}}\right)
$$

and

$$
\gamma_{i}^{\prime}=\frac{1}{\sqrt{\left.\alpha_{i}^{2}-\beta_{i\left(V_{i(r)(T)}^{\prime}\right)}^{2}\right)}}
$$

The absolute position four-vector, $P 4_{i(r e s t)_{-}(a b s)}$, can be turned into an arbitrary relativistic four vector by changing $X_{i(0)}$ into an arbitrary position, $X_{i(0) \_(a r b)}$ such:

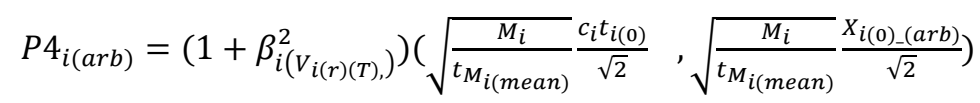

and $P 4_{i(a r b)}$ should transform similarly to $P 4_{i(r e s t)_{-}(a b s)}$ according to equation 19 , only $V_{r(T)}$ is exchanged in $V_{r}$ in the expression for $\gamma_{i}^{\prime}$ and $\beta_{i}^{\prime}$ in the transformation matrix. Therefore, the decay time $t_{i(0)}$, of a particle moving at velocity $V_{r}$ from the lab, should be measured at the lab as, $t_{i(0)}^{\prime}$, such:

$$
t_{i(0)}^{\prime}=\frac{\left(1+\beta_{i\left(V_{i(r)(T),}^{2}\right)}\right)}{\left(1+\beta_{i\left(V_{i(r)(T)}^{\prime}\right)}^{\prime}\right)} \gamma_{i} t_{i(0)}
$$

Where $\gamma_{i}$ is given in equation $7 \mathrm{~b}$.

\section{Absolute Energy at Rest}

The particle's absolute energy at rest may be defined from the already defined expressions for $E_{i(a b s)}$ and $P_{i(a b s)}$. Therefore, we may define an absolute energy-momentum four vector such:

$$
E 4_{i(a b s)}=\left(\frac{E_{i(a b s)}}{c_{i\left(v_{i(r)(T)}\right)}}, P_{i(a b s)}\right)
$$

The length of $E 4_{i(a b s)}$ should give us particle's i absolute rest energy, $E_{i(r e s t)}$ :

From equations 11 b,c we may have:

$$
E_{i(a b s)}^{2}-c_{i\left(V_{r(i)(T)}^{2}\right.}^{2} P_{i(a b s)}^{2}=E_{i(r e s t)}^{2}
$$




$$
\begin{aligned}
& E_{i(a b s)}=\frac{M_{i}}{t_{M_{i(\text { mean })}}}\left(c_{i}^{2} t_{i(a b s)}\right) \\
& \boldsymbol{P}_{\boldsymbol{i ( a b s )}}=\frac{M_{i} V_{i(r)(T)}}{t_{M_{i(\text { mean })}}} t_{i(a b s)}
\end{aligned}
$$

Therefore:

$$
\begin{aligned}
& \left(\frac{M_{i} c_{i\left(V_{r(i)(T)}^{2}\right.}}{t_{M_{i(\text { mean })}}} \gamma_{i} t_{i}\right)^{2}-c_{i\left(V_{r(i)(T)}^{2}\right.}\left(\frac{M_{i} V_{i(r)(T)} \gamma_{i} t_{i}}{t_{M_{i(\text { mean })}}}\right)^{2} \\
& \frac{M_{i}^{2} c_{i\left(V_{r(i)(T)}\right.}^{4}}{t_{M_{i(\text { mean })}}^{2}} \gamma_{i}^{2} t_{i}^{2}-\frac{c_{i\left(V_{r(i)(T)}\right.}^{2} M_{i}^{2} V_{i(r)(T)}^{2}}{t_{M_{i(\text { mean })}^{2}}^{2}} \gamma_{i}^{2} t_{i}^{2} \\
& \frac{M_{i}^{2} c_{i\left(V_{r(i)(T)}\right.}^{2}}{t_{M_{i(\text { mean })}}^{2}} \gamma_{i}^{2} t_{i}^{2}\left(c_{i\left(V_{r(i)(T)}\right.}^{2}-V_{i(r)(T)}^{2}\right)
\end{aligned}
$$

But since from equation $7 \mathrm{~d}, \gamma_{i}^{2}=\frac{1}{\left(\frac{\left.c_{i\left(V_{r(i)(T)}\right.}^{2}-\frac{V_{i(r)(T)}^{2}}{c_{i(0)}^{2}}\right)}{c_{i(0)}^{2}}\right)}$ we can write:

$$
\frac{M_{i}^{2} c_{i\left(V_{r(i)(T)}^{2}\right.}}{t_{M_{i(\text { mean })}}^{2}} \gamma_{i}^{2} t_{i}^{2} c_{i(0)}^{2}
$$

Therefore:

$$
E_{i(\text { rest })}=\frac{M_{i} c_{i(0)} c_{i\left(V_{i(r)(T)}\right)}}{t_{M_{i(\text { mean })}}} t_{i}
$$

Using equation 15 we may substitute for $t_{i}$ :

$$
E_{i(\text { rest })}=\frac{M_{i} c_{i(0)} c_{i\left(V_{i(r)(T)}\right)}}{t_{M_{i(\text { mean })}}}\left(1+\beta_{i}^{2}\right) t_{i(0)}
$$

In order that $E_{i(r e s t)}$ wouldn't depend on velocity:

$$
\begin{aligned}
& c_{i\left(V_{i(r)(T)}\right)}\left(1+\beta_{i}^{2}\right)=c_{i(0)} \\
& c_{i\left(V_{i(r)(T)}\right)}=\frac{c_{i(0)}}{\left(1+\beta_{i}^{2}\right)}
\end{aligned}
$$

Therefore:

$$
E_{i(\text { rest })}=\frac{M_{i} c_{i(0)}^{2}}{t_{M_{i(\text { mean })}}} t_{i(0)}
$$

The above leads us to redefine the particle's absolute position that doesn't depend on velocity, i.e. $P_{i(r e s t)_{-}(a b s)}$, and modify equation 5a to be:

$$
P_{i(\text { rest })_{-}(a b s)}=\frac{M_{i}}{t_{M_{i(\text { mean })}}}\left(\frac{\left(V_{r(i)(T)} \gamma_{i} t_{i(0)}\right)^{2}}{2}-\frac{\left(c_{\left.i\left(v_{i(r)(T)}\right)_{i} t_{i(0)}\right)^{2}}\right)}{2}\right)
$$

Which gives for particle $\mathrm{i}$, using equation $4 \mathrm{~b}$, an absolute position of:

$$
P_{i(r e s t)_{-}(a b s)}=\frac{M_{i}}{t_{M_{i(\text { mean })}}}\left(c_{i(0)}^{\prime} t_{i(0)}\right)^{2}
$$




\section{Experimental Implications}

\subsection{Energy Implications}

If the above is correct, there should be experimental implications for it. Therefore, the modified expressions to the energy and momentum of a particle, $E_{i(a b s)}, P_{i(a b s)}$ should be manifested experimentaly.

Let us focus at the energy expression, $E_{i(a b s)}$ shown in equation 11c. As we can see, the dependence of $E_{i(a b s)}$ on the particles velocity is different than the known expression given in special relativity:

$$
E_{i(s r)}=\gamma_{i(s r)} M_{i} c^{2}
$$

Where $\gamma_{i(s r)}=\frac{1}{\sqrt{1-\frac{v_{i(r)}^{2}}{c^{2}}}}$

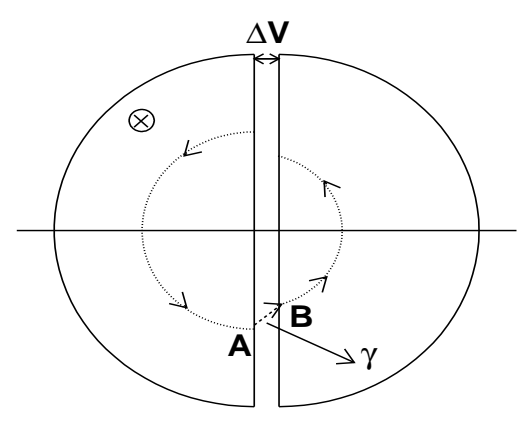

Figure 2. A particle moving in a cyclotron. In this setup the particle is moving against the direction of an electric field present between the two cyclotron's plates which are held at a voltage difference, $\Delta V$. Therefore, the particle will lose energy as it moves between points $\mathrm{A}$ and $\mathrm{B}$ and releases a photon, $\gamma$.

Therefore, we may attempt to use this possible different velocity dependence in order to attempt and reveal a more accurate expression for the particle's energy.

To do that, let us consider the experimental setup shown in Figure 2 (Halzen \& Martin, 1984), in which a particle is initially moving in a circular motion at the larger half circle with velocity $V_{r 1}$. As the particle reaches point A, it is subjected to an electric field in an opposite direction to its direction of motion. Therefore, the particle will slow down to velocity, $V_{r 2}$, and continue to move in a smaller circular motion from point $\mathrm{B}$ onwards. In the process of slowing down due to the electric field, the particle is expected to emit a photon $\gamma$, with energy, $E_{i(\gamma)}$, that may be detected by photon detectors surrounding the cyclotron (not shown). As we can see from equations 11c and 26, a particle with an initial and final velocities $V_{r 1}, V_{r 2}$, may have two possible sets of initial and final energies. Therefore, the energy difference, which is manifested in the energy of the photon, should also be different in the two possible energy cases. i.e. measuring the photon's energy while knowing exactly the initial and final velocities $V_{r 1}, V_{r 2}$, may help us determine which of the energy expressions, $E_{i(s r)}$ or $E_{i(a b s)}$ is more correct. 
The above experiment may be repeated many times with different particles and different velocities in order to produce a distribution for the photon energy values which may allow a careful investigation of the above.

\subsection{Lifetime Implications}

The suggested dependence of $t_{i}$ on the particle velocity $V_{r(T)}$ should clearly have an effect on the particle mean lifetime measurement. As we know from the Standard Model, the mean lifetime measurement depends on the particle's charge. Thus it should be affected from the running coupling constant effect (Halzen \& Martin, 1984). Therefore, combining the above, we may suggest that $t_{i}{ }^{\prime} s$ possible dependence on $V_{r(T)}$ may be a part of the description of the running coupling constant effect. This is in fact manifested in the expression for the particle's charge given in Brodet (2017b) and also from equation $\mathbf{1 8 b}$ where there is a dependence of $Q_{i(T)_{-}(a b s)}$ on $t_{i}$. From the experimental point of view, the running coupling effect is known to be related with energy. It is suggested here to investigate this effect more specifically and attempt to measure the running coupling constant as a function of the particles velocity. i.e. to base the measurement of this effect on a direct measurement of the particle's velocity rather than energy, using detectors as Cherenkov detectors (Arnold et al., 1988). This may allow us to test if the expression given here for $Q_{i(T)_{-}(a b s)}$ describes the running coupling constant effect correctly or not.

\section{Conclusions}

The absolute position of a particle was discussed in terms of two separate definitions. The first definition describes the particle's absolute position at rest and the second describes an absolute position containing also a contribution from the particle's velocity with respect to the expanding universe. Furthermore, a definition for the particle's absolute energy was calculated from the particle's absolute position. Moreover, from the calculation of the particle's absolute energy, it was possible to identify a dependence of the particle's decay time in its own rest frame on the particle's velocity with respect to the expanding universe. Consequently, the above was related to the particle mean lifetime and was discussed in the context of the running coupling constant and the possible mean lifetime dependence on the particle's velocity. Finally, experimental ways to investigate and test the above were presented.

\section{References}

Arnold, R., Baillon, P., Besch, H. J., Bosteels, M., Christophel, E., Dracos, M., ... \& Séguinot, J. (1988). A ring imaging Cherenkov detector, the DELPHI Barrel RICH Prototype: Part A: Experimental studies of the detection efficiency and the spatial resolution. Nuclear Instruments and Methods in Physics Research Section A: Accelerators, Spectrometers, Detectors and Associated Equipment, 270(2-3), 255-288. https://doi.org/10. 1016/0168-9002(88)90695-X

Brodet, E. (2010). The possibility of a hidden variable in time. Physics Essays, 23(4).

Brodet, E. (2012). A new look at the transition between potential and kinetic energy. Physics Essays, 25(1).

Brodet, E. (2013). Hypothesized lepton inner discreteness. Physics Essays, 26(2), 247-250.

Brodet, E. (2016). The Relationship Between the Possibility of a Hidden Variable in Time, Possible Photon Mass, Particle's Energy, Momentum and Special Relativity. Applied Physics Research, 8(6), 13. http://dx.doi.org/10.5539/apr.v8n6p13

Brodet, E. (2017a). The Total Energy and Momentum Stored in a Particle. Applied Physics Research, 9(2), 65. https://doi.org/10.5539/apr.v9n2p65

Brodet, E. (2017b). Possible hidden symmetry in time. Physics Essays, 30(4), 383-387.

Brodet, E. (2018). The distribution of fundamental particles' absolute position. Physics Essays, 31(1), 39-42.

Halzen, F., \& Martin, A. D. (1984). Quarks and leptons. New York: Wiley.

\section{Copyrights}

Copyright for this article is retained by the author(s), with first publication rights granted to the journal.

This is an open-access article distributed under the terms and conditions of the Creative Commons Attribution license (http://creativecommons.org/licenses/by/4.0/). 